\title{
CRECIMIENTO Y DESARROLLO DE ALBAHACA (Ocimum basilicum L.) BAJO CUBIERTA EN LA SABANA DE BOGOTÁ
}

\section{GROWTH AND DEVELOPMENT OF BASIL (Ocimum basilicum L.) UNDER COVER IN THE SABANA OF BOGOTÁ}

\author{
Fernando Colorado ${ }^{1}$, Imelda Montañez ${ }^{2}$, Catalina Bolaños ${ }^{3}$ Jorge Rey $^{4}$
}

\begin{abstract}
${ }^{1}$ M.Sc. Profesor titular. Facultad de Ingeniería Agronómica Universidad de Ciencias Aplicadas y Ambientales, U.D.C.A, Bogotá, fcolorad@udca.edu.co; ${ }^{2}$ M.Sc. Profesora Facultad de Ingeniería Agronómica Universidad de Ciencias Aplicadas y Ambientales U.D.C.A, Bogotá, bmontañez@udca.edu.co; ${ }^{3}$ Estudiante Ingeniería Agronómica U.D.C.A, ktkhermosa@gmail. com; ${ }^{4}$ Estudiante Ingeniería Agronómica U.D.C.A bbscolombia@gmail.com
\end{abstract}

Rev. U.D.C.A Act. \& Div. Cient. 16(1): 121 - 129, 2013

\section{RESUMEN}

El desarrollo de la albahaca puede ser establecido y cuantificado, mediante la obtención de parámetros de crecimiento y con la caracterización de sus fases de desarrollo, información útil para el manejo agronómico y comercial. En el presente trabajo, se buscó obtener la TCR, el IAF y la TAN de esta especie, sembrada bajo cubierta; igualmente, determinar sus fases de desarrollo. El ensayo, se realizó en la U.D.C.A, entre el 2010 y el 2011 , en un lote de $70 \mathrm{~m}^{2}$, con la especie Ocimum basilicum L. Se empleó una densidad de población de 12 plantas por $\mathrm{m}^{2}$. Se midió altura, número de hojas, área foliar y peso seco. La lectura se hizo cada cuatro días. El ensayo, se llevó hasta el inicio de la floración: 61ddt. Los parámetros de crecimiento, se estipularon empleando el enfoque funcional del modelo logístico, con el paquete estadístico SAS. A partir de la información analizada, se determinó la eficiencia fisiológica. Las fases de desarrollo, se caracterizaron en el mismo período de tiempo y se estimaron contabilizando los días trascurridos entre eventos fisiológicos de la planta o cambios observables en las variables de estudio y determinando la eficiencia fisiológica por fase. Se establecieron tres fases de desarrollo y las tasas de crecimiento se especificaron, obteniendo valores máximos de TCR de 0,1082 $\mathrm{g} \mathrm{g}^{-1} \mathrm{~d}^{-1}$, a los 5ddt; TAN de 0,0083 $\mathrm{g} \mathrm{cm}^{2}$ $\mathrm{d}^{-1}$, al finalizar los $14 \mathrm{ddt}$ e IAF de 0,047, a los 31ddt.

Palabras clave: Eficiencia fisiologica albahaca, planta condimentaria, manejo agronómico.

\section{SUMMARY}

The development of basil can be established and quantified by obtaining growth parameters and the characterization of the developmental phases, useful information for its commercial and agricultural management. In this paper it was sought to obtain the TCR, the IAF and the TAN of the species, planted under cover, also to determine their stages of development. The research was conducted in the U.D.C.A between 2010 and 2011 in an area of $70 \mathrm{~m}^{2}$ with the species Ocimum basilicum L. A population density of 12 plants per $\mathrm{m}^{2}$ was used. Height, number of leaves, leaf area and dry weight was measured. The counts were made every four days. The trial was carried up to the start of flowering: $61 \mathrm{ddt}$. Growth parameters were determined using the functional approach of the logistic model with the SAS statistical package. From the analyzed information the physiological efficiency was determined. The development phases were characterized in the same period and were estimated by counting the days elapsed between plant physiological events or observable changes in the variables and determining the physiological efficiency per phase. Three phases of development were identified and growth rates were specified, obtaining maximum TCR values of $0.1082 \mathrm{~g} \mathrm{~g}^{-1} \mathrm{~d}^{-1}$ at $5 \mathrm{ddt}$; TAN of $0.0083 \mathrm{~g} \mathrm{~cm}^{2} \mathrm{~d}^{-1}$ at the end of the 14ddt and IAF of 0.047 at 31ddt.

Key words: Basil physiology efficiency, spice plant, agronomic management. 


\section{INTRODUCCIÓN}

La albahaca (Ocimum basilicum L.) es una de las plantas aromáticas más apetecidas en el ámbito culinario, por tener un sabor dulce y fragante. Para obtener hojas con altas concentraciones de aceites esenciales, se deben colectar poco antes de la floración, ya que contienen una mayor cantidad de sustancias oleosas, que determinan su aroma; las hojas adultas, se caracterizan por tener un sabor picante (CCI, 2007). Originaria de la India y es empleada como especia y condimento alimentario. Existe una variedad de hoja pequeña, denominada albahaca simplemente y otra de hoja ancha, de mayor uso gastronómico, llamada más comúnmente basílico (Berdonces, 2010). De la anterior, se conocen la variedad genovese y el hibrido nufar, de porte más vigoroso y de mayor productividad que la genovese. Puede llegar a producir entre 60 y $70 \mathrm{t} \mathrm{ha}^{-1}$ de hoja en fresco y se cultiva en los departamentos de Boyacá, Valle, Cundinamarca y Antioquia (CCI, 2007).

El crecimiento de una planta es un incremento irreversible de tamaño, de peso seco y de cantidad de citoplasma, los cuales, son cambios cuantitativos. El proceso de desarrollo está constituido por los cambios de forma, así como por el grado de diferenciación y de complejidad, que alcanza cada organismo, como son la aparición de flores, de frutos o de semillas.

El crecimiento es una aproximación holística, explicativa e integral, para comprender la forma y las funciones de los vegetales (Hunt, 2003). En cualquier sistema biológico, el crecimiento es la consecuencia de la división celular (incremento en número) y de la elongación celular (aumento en tamaño) (Casierra \& Cardozo, 2009)

Las curvas de crecimiento en los vegetales son un reflejo del comportamiento de una planta en un ecosistema particular con respecto al tiempo (Casierra-Posada et al. 2003). Su elaboración permite conocer el crecimiento de una planta, a través del tiempo; curva que varía en función de variables propias de la especie y del ambiente donde crece.

Hunt (2003) afirma que. por lo general, los análisis de crecimiento se hacen con materia seca y que las plantas anuales y perennes que crecen bajo condiciones normales han mostrado, en sus primeros estados, la misma curva típica de crecimiento.

Abello et al. (2006) aseveran que el crecimiento es un proceso irreversible derivado de un aumento en el tamaño y es el resultado de la división de la célula y de su elongación.

Al representar el crecimiento en función del tiempo, se obtiene una curva sigmoidea, denominada curva de crecimiento. La zona de crecimiento lento responde al proceso inicial en que no existen muchos centros meristemáticos y la incorporación de masa, a través de la fotosíntesis es pequeña. A medida que en la planta joven aumenta la masa se produce un aumento de células meristemáticos y de área fotosintética y, por tanto, el potencial de crecimiento se hace mayor y entran así en la zona de crecimiento rápido (Barceló et al. 1995). Salisbury \& Ross (1994) afirman que la curva sigmoidea está representada por tres fases: logarítmica, lineal y de senescencia.

El conocimiento de la fenología en diferentes fechas de siembra y en distintas condiciones agroecológicas constituye uno de los aspectos agronómicos fundamentales para el cultivo y la producción de cualquier vegetal. El registro de los diferentes eventos fisiológicos que determinan las fases fenológicas de las plantas permiten al investigador y productor relacionar el comportamiento del cultivo con el clima y el manejo de las plantas (Barroso \& Jerez, 2002). Los citados investigadores, trabajando en un suelo ferrolítico de La Habana y a diferentes épocas de siembra de albahaca blanca caracterizaron seis fases fenológicas del cultivo, desde siembra y durante 76 días hasta la senescencia del primer par de hojas verdaderas.

Ghazi et al. (2012) señalan cuatro etapas de desarrollo en albahaca trabajando en Irán con dos variedades de la especie (blanca y morada), diferentes arreglos de siembra y diferente población: una primera etapa, va desde siembra hasta que la planta posee entre 4 y 6 hojas verdaderas; la segunda, se extiende hasta floración; la producción de semilla caracteriza el inicio de la tercera etapa y la madurez de la planta, la ubica en la cuarta.

Basado en lo anterior, esta investigación planteó obtener la tasa de crecimiento del cultivo (TCR), el índice de área foliar (IAF) y la tasa de asimilación neta (TAN), como parámetros de crecimiento de la albahaca (O. basilicum L.); igualmente, caracterizar sus fases de desarrollo bajo condiciones de invernadero, en la Sabana de Bogotá.

\section{MATERIALES Y MÉTODOS}

El ensayo, se llevó a cabo en la Universidad de Ciencias Aplicadas y Ambientales U.D.C.A, en Bogotá D.C, entre el segundo semestre de 2010 y el primer semestre de 2011, a una altitud de $2540 \mathrm{msnm}$, con las siguientes condiciones de invernadero: temperaturas mínimas en las horas de la mañana, entre $2^{\circ} \mathrm{C}$ y $5^{\circ} \mathrm{C}$; temperatura al mediodía, entre los $25^{\circ} \mathrm{C}$ y los $29^{\circ} \mathrm{C}$ y temperaturas máximas alrededor de las 3:00 p.m., cercanas a los $38^{\circ} \mathrm{C}$. La humedad relativa fluctuaba al mediodía, entre el $45 \%$ y $62 \%$, llegando al $98 \%$, como máxima y, entre el $15 \%$ y $20 \%$, como mínima. El lote destinado para el ensayo tuvo un área de $70 \mathrm{~m}^{2}$. Para conocer 
las condiciones físicas y químicas del suelo, se tomó una muestra de suelo al iniciar los ciclos de siembra; se rotuló y se envió al laboratorio, para la caracterización de elementos mayores y menores.

El material vegetal utilizado fue de la especie $O$. basilicum L. variedad genovese. Para la siembra, se utilizó una plantilla según la especie, llegando a una densidad de población de doce plantas por $\mathrm{m}^{2}$. El ensayo se manejó agronómicamente efectuando labores de deshierbas, riegos, aporques, según necesidad del cultivo; no se aplicaron sustancias orgánicas o químicas para la protección de cultivos. El trabajo evalúo las siguientes variables: altura de planta, número de hojas, área foliar y peso seco de la planta completa.

Para tomar la información correspondiente de las variables de estudio, se identificó el $20 \%$ de la población de plantas en campo, de forma aleatoria. La lectura de las variables a evaluar se hizo cada cuatro días. El ensayo, se llevó hasta el inicio de la floración, bajo las condiciones anteriormente descritas: 61 días después de transplante. El registro de datos fue de la siguiente forma: peso seco: una vez tomado el dato de peso fresco de la planta, el material vegetal se depositó en bolsas de papel para ser secadas en una estufa de $400 \mathrm{~kg}$ de capacidad, a temperatura de $70^{\circ} \mathrm{C}$, durante 48 horas. Área foliar: se determinó empleando la metodología del $\mathrm{cm}^{2}$, que consiste en tomar un centímetro cuadrado de hoja, pesarlo y extrapolar al peso total de las hojas. Altura: se midió en $\mathrm{cm}$ desde la base del tallo hasta su ápice. Número de hojas: con conteo manual de hojas verdaderas.

Como parámetros de crecimiento, se tomaron la tasa de asimilación neta (TAN), la tasa relativa de crecimiento (TRC) y el índice de área foliar (IAF). Estos parámetros, se determinaron empleando el enfoque funcional del modelo logístico, donde la curva de crecimiento se define por la expresión matemática:

$y=\alpha / 1+e^{-k(t-y)}$

donde $\alpha$ la asíntota superior, es el máximo tamaño, es decir, $y=\alpha$ cuando $t \rightarrow \infty$ y $y=0$ cuando $x \rightarrow-\infty$ es la asíntota inferior $y$ es el tiempo en que se logra la máxima tasa de crecimiento (Flórez et al. 2006).

Con base en estos coeficientes obtenidos por el paquete estadístico SAS, se construyó la curva de crecimiento. A partir de la información analizada, se determinó la eficiencia fisiológica. Las fases de desarrollo se caracterizaron desde el momento del transplante hasta el momento de inicio de la floración y se estimaron contabilizando los días trascurridos entre eventos fisiológicos de la planta o cambios observables en las variables de estudio y determinando la eficiencia fisiológica por fase.

\section{RESULTADOS Y DISCUSIÓN}

De acuerdo con los resultados del análisis químico el suelo donde se realizó la investigación es ácido $(\mathrm{pH} 5,4)$ y presenta valores altos en cuanto a materia orgánica $(14,3 \%)$, fósforo disponible $\left(266 \mathrm{mg} \mathrm{kg}^{-1}\right)$, calcio $\left(14,3 \mathrm{cmol}(+) \mathrm{kg}^{-1}\right)$, magnesio $\left(4,3 \mathrm{cmol}(+) \mathrm{kg}^{-1}\right)$, azufre $\left(126 \mathrm{mg} \mathrm{kg}^{-1}\right)$, hierro $\left(149 \mathrm{mg} \mathrm{kg}^{-1}\right) \mathrm{y}$ zinc $\left(29,4 \mathrm{mg} \mathrm{kg}^{-1}\right)$. Igualmente, la capacidad de intercambio catiónica es alta $\left(53,1 \mathrm{cmol}(+) \mathrm{kg}^{-1}\right)$. En el caso del potasio $\left(0,33 \mathrm{cmol}(+) \mathrm{kg}^{-1}\right)$, cobre $\left(2,0 \mathrm{mg} \mathrm{kg}^{-1}\right)$ y boro $\left(0,24 \mathrm{mg} \mathrm{kg}^{-1}\right)$, su contenido en el suelo está en nivel medio, lo mismo que el porcentaje de saturación de bases $(37,9 \%)$. La relación calcio: magnesio es óptima $(3,32: 1)$ y el elemento que se encuentra bajo es el manganeso $\left(3,6 \mathrm{mg} \mathrm{kg}^{-1}\right)$. También se presenta bajo el porcentaje de saturación de aluminio $(2,1 \%)$, lo cual, es importante, dado que la toxicidad de aluminio es el principal factor limitante del crecimiento de las plantas en los suelos ácidos (Dong et al. 2002).

El contenido alto de materia orgánica representa una cualidad importante en este suelo, teniendo en cuenta que las condiciones de temperatura bajo cubierta favorecen la mineralización de la misma y, con ello, se incrementa la disponibilidad de algunos elementos nutricionales esenciales para el cultivo, como el nitrógeno, el fósforo y el potasio (Matus \& Maire, 2000).

Los resultados del análisis indican que el suelo empleado en la investigación presenta buena disponibilidad de nutrientes esenciales para la planta, por su buena saturación de bases, contenido de materia orgánica y niveles adecuados de elementos mayores.

Materia seca: El contenido de materia seca se fue incrementando paulatinamente hasta obtener valores de $14,07 \mathrm{~g}$ a los 61 días después de trasplante (ddt), presentándose un comportamiento típico en el crecimiento, como se ilustra en la gráfica $1 \mathrm{~A}$, donde se observa la curva sigmoidea La expresión matemática empleada para definir la curva de crecimiento tuvo un buen ajuste al obtener $\mathrm{R}^{2}$ de 0,99. Carranza et al. (2009) llegaron a una situación similar en plantas de lechuga sembradas en un suelo salino de la Sabana de Bogotá.

Área foliar: De forma similar al contenido de materia seca, el área foliar se fue incrementando hasta obtener valores de $132,6 \mathrm{~cm}^{2}$ a los 61 días después de trasplante (gráfica 1B). La tasa de crecimiento de las hojas depende de la masiva e irreversible expansión de células jóvenes, las cuales, son producidas por la división celular en los tejidos meristemáticos (Carranza et al. 2009). El área foliar total, que ha sido directamente relacionada con la cantidad de clorofila, es un parámetro importante para estimar la habilidad de la planta para sintetizar materia seca (Campostrini \& Yamanishi, 
2001). Se presentó una fase lenta de crecimiento de área foliar, que coincide con la baja acumulación de materia seca en los primeros 19 días después de trasplante (Gráficas 1A, 1B). En un trabajo en papa realizado por Aguilar et al. (2006) en Metepec, México, midiendo crecimiento de dos variedades, se encontró un comportamiento similar, donde la etapa lenta de crecimiento duró dos semanas. La curva descrita en la gráficas 1B obedece a un buen ajuste, obtenido con la expresión matemática con un $\mathrm{R}^{2}$ de 0,99 ; quiere decir esto, que el crecimiento de la albahaca se asemeja a la curva sigmoide, expresada mediante la ecuación matemática planteada; sin embargo, es de resaltar el hecho que el área foliar obtenida es baja si se compara con resultados hallados en otros trabajos; es así que Contreras \& Gómez (2008), trabajando en Honduras con tres variedades de albahaca, nufar, genovese y aromaz, en dos sistemas de producción, suelo e hidroponía, encontraron valores de área foliar de $901,4 \mathrm{~cm}^{2}$ y $2002,7 \mathrm{~cm}^{2}$ para hidroponía y suelo, respectivamente.
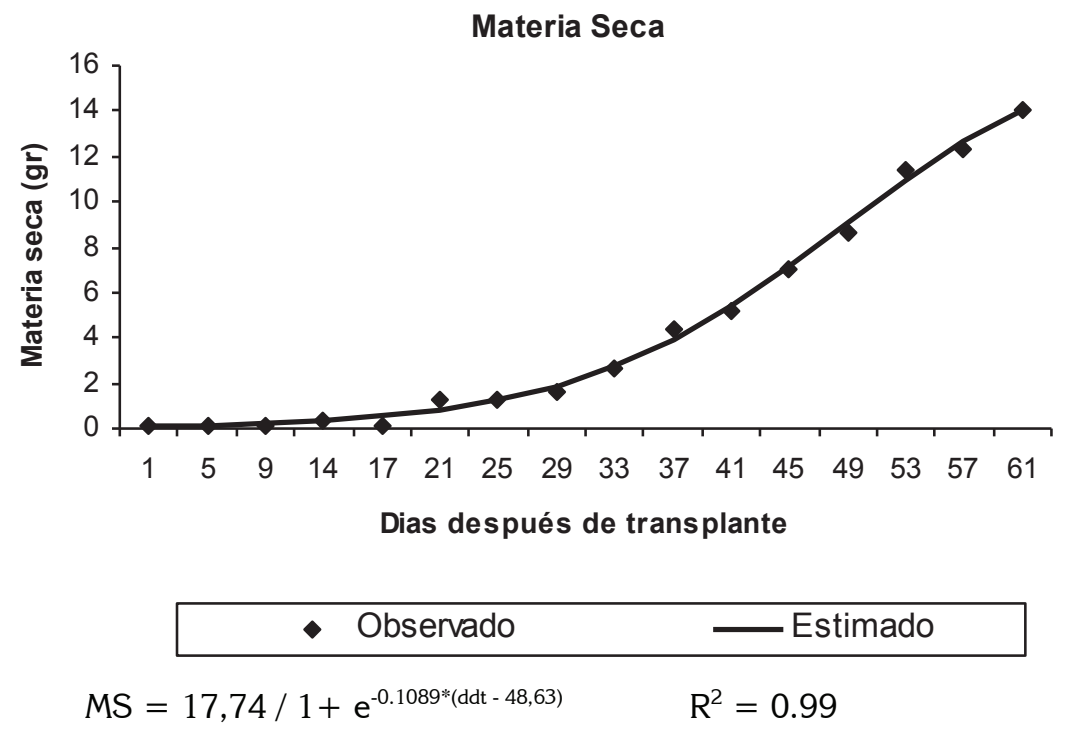

A.

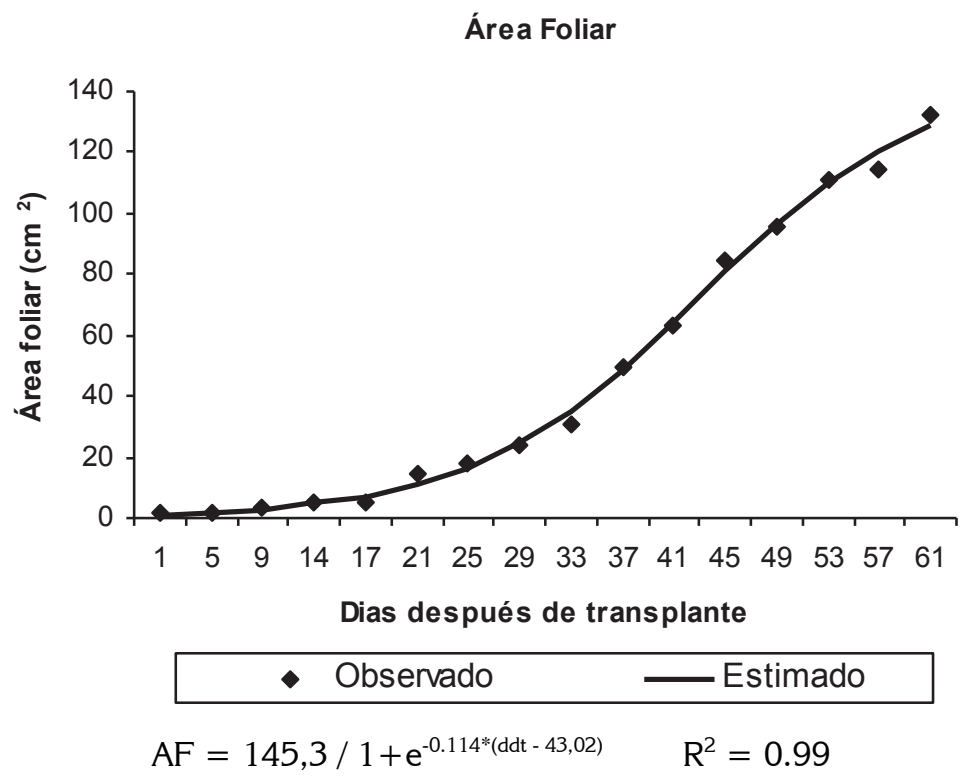

B.

Gráfica 1. Comportamiento de la materia seca (A) y área foliar (B) de plantas de albahaca incluyendo la modelación empleada. 
Altura y número de hojas: La albahaca incrementó su altura en $35,5 \mathrm{~cm}$, al pasar de $2 \mathrm{~cm}$ al momento del trasplante a $37,5 \mathrm{~cm}$, al finalizar el ensayo; en el mismo periodo de tiempo, pasó de 3 a 45 hojas (Tabla 1). Aunque estas variables no son componentes de los parámetros de crecimiento, sí resulta importante su cualificación, pues son determinantes a la hora del manejo comercial del cultivo, ya que en esta especie se cosechan sus hojas en varios cortes. La altura de la planta tuvo un buen comportamiento para las condiciones del trabajo, puesto que en clima cálido, Ramírez et al. (2001) reportan una altura promedio de $28,7 \mathrm{~cm}$ trabajando con siete variedades de albahaca, en zona cafetera colombiana. Al respecto, Castro (2005) afirma que temperaturas entre $24^{\circ} \mathrm{C}$ y $30^{\circ} \mathrm{C}$, durante el día y $16^{\circ} \mathrm{C}$ y $20^{\circ} \mathrm{C}$, en la noche, combinados con una longitud del día de $16 \mathrm{~h}$, inducen una alta tasa de desarrollo.

Tabla 1. Fases de desarrollo de albahaca.

\begin{tabular}{|c|c|c|c|c|c|c|c|c|c|c|c|c|}
\hline \multicolumn{2}{|c|}{ Fase } & \multicolumn{2}{|c|}{ Altura $(\mathrm{cm})$} & \multicolumn{2}{|c|}{ Número de hojas } & \multicolumn{2}{|c|}{ Peso (g) } & \multicolumn{2}{|c|}{ Área foliar $\left(\mathrm{cm}^{2}\right)$} & \multicolumn{3}{|c|}{ Tasas de crecimiento } \\
\hline & DDT & $\begin{array}{c}\text { Variación } \\
\text { total }\end{array}$ & $\begin{array}{c}\text { Variación } \\
\text { / día }\end{array}$ & $\begin{array}{l}\text { Variación } \\
\text { total }\end{array}$ & $\begin{array}{c}\text { Variación } \\
\text { / día }\end{array}$ & $\begin{array}{l}\text { Variación } \\
\text { total }\end{array}$ & $\begin{array}{c}\text { Variación } \\
\text { / día }\end{array}$ & $\begin{array}{l}\text { Variación } \\
\text { total }\end{array}$ & $\begin{array}{c}\text { Var0iación } \\
\text { / día }\end{array}$ & $\begin{array}{c}\text { TRC } \\
\left(\mathrm{g} \mathrm{g}^{-1} \mathrm{~d}^{-1}\right)\end{array}$ & $\begin{array}{l}\text { TAN } \\
\underset{\left(\mathrm{g} \mathrm{cm}^{-1)}\right.}{ }\end{array}$ & IAF \\
\hline 1 & $0-14$ & $2-4,6$ & 0,18 & $3-7$ & 0,29 & $0,08-0,3$ & 0,062 & $2-4,8$ & 0,2 & 0,1064 & 0,0083 & 0,0082 \\
\hline 2 & $14-31$ & $4,6-9$ & 0,35 & $7-12$ & 0,32 & $0,3-2,63$ & 0,62 & $4,8-31,1$ & 1,64 & 0,092 & 0,0071 & 0,047 \\
\hline 3 & $31-61$ & $9-37,5$ & 1,14 & $12-45$ & 1,12 & $2,63-14,07$ & 2,82 & $\begin{array}{l}31,1- \\
132,6\end{array}$ & 4,8 & 0,022 & 0,0024 & 0,206 \\
\hline
\end{tabular}

Tasas de crecimiento: El crecimiento de la albahaca expresado con las tasas de crecimiento tuvo un comportamiento típico en cuanto a la secuencia de eventos fisiológicos, dados en las tres fases de desarrollo cualificadas, es decir, crecimiento lento, lineal y exponencial, durante el período de tiempo analizado. Las plantas manifiestan cambios en características de desarrollo y de estructura, debido a su exposición en ambientes particulares (Grime \& Mackey, 2002).

Tasa relativa de crecimiento (TRC): La TRC máxima se alcanzó en los primeros cinco días, con $0,1082 \mathrm{~g} \mathrm{~g}^{-1} \mathrm{~d}^{-1}$, reduciéndose a $0,1064 \mathrm{~g} \mathrm{~g}^{-1} \mathrm{~d}^{-1}$, al finalizar el día 14 , después de trasplante (ddt); posteriormente, entre el día 15 y el día 31 llegó a una tasa de $0,092 \mathrm{~g} \mathrm{~g}^{-1} \mathrm{~d}^{-1}$, para finalizar a los 61ddt, con una tasa de $0,022 \mathrm{~g} \mathrm{~g}^{-1} \mathrm{~d}^{-1}$ (Tabla 1). Baracaldo et al. (2010), trabajando con la variedad Nelson en clavel, identificaron un comportamiento similar en esta tasa, argumentando que de acuerdo a Brown (1984), el máximo valor ocurre al principio y luego decrece con la importación de asimilados a diferentes órganos de la planta. Barreiro et al. (2006) encontraron una TCR de $0,08 \mathrm{~g} \mathrm{~g}^{-1} \mathrm{~d}^{-1}$, a los 50 días después de siembra (dds), para plantas de albahaca tratadas con agua en presiembra; la tasa decreció casi hasta $0 \mathrm{~g} \mathrm{~g}^{-1} \mathrm{~d}^{-1}$, a los 106dds.

Índice de área foliar (IAF): De lento crecimiento durante los primeros 31ddt, la tasa alcanzó un valor de 0,047, durante este período y finalizó en 0,206, a los 61 ddt (gráfica 2B). El índice es bajo, como consecuencia de un área foliar reducida, que se traduce en una baja capacidad para interceptar la radiación solar. Maldonado \& Corchuelo (1993) reportan un IAF, de 2,88 y 3,1, para las variedades cerinza y tundama de frijol, a los 64dds; aunque Barraza et al. (2004), trabajando con tomate, variando densidades de población, hallaron un IAF cercano al 0,5, a los 60dds y de 4,1, a los 105dds. Los resultados anteriores para albahaca son importantes, ya que Ruíz et al (2008), ensayando con la especie en Baja California, México, encontraron una relación positiva ( $r=$ 0,49 ), entre el IAF y el rendimiento agrícola obtenido, que decrece en la medida que el IAF sea bajo.

Tasa de asimilación neta (TAN): El comportamiento de esta tasa fue decreciente a lo largo del período de tiempo evaluado, teniendo un valor de $0,0083 \mathrm{~g} \mathrm{~cm}^{2} \mathrm{~d}^{-1}$, al finalizar los $14 \mathrm{ddt}$; posteriormente, al finalizar los $31 \mathrm{ddt}$ presentó un valor de $0,0071 \mathrm{~g} \mathrm{~cm}^{2} \mathrm{~d}^{-1}$, para finalizar a los $61 \mathrm{ddt}$, en $0,0024 \mathrm{~g} \mathrm{~cm}^{2}$ $\mathrm{d}^{-1}$ (gráfica 2C). Barreiro et al. (2006) determinaron una TAN máxima para albahaca, a los $64 \mathrm{dds}$, de $0,005 \mathrm{~g} \mathrm{~cm}^{2} \mathrm{~d}^{-1}$. La capacidad de la planta para generar fotoasimilados está en función del área foliar, de la disposición y edad de las hojas y de los procesos de regulación interna, relacionados con la demanda de asimilados (Hunt, 1982); igualmente, los autores afirman que la TAN decrece con el desarrollo del cultivo a lo largo del ciclo de producción. Jarma et al. (2006) reportaron valores de TAN para estevia, cercanos a $0,001 \mathrm{~g}$ $\mathrm{cm}^{2} \mathrm{~d}^{-1}$, a los $60 \mathrm{dds}$, con un $100 \%$ de radiación incidente. La respuesta en este parámetro es consistente a juicio de Hunt (1982), quien indica que esta tasa puede ser modificada por fluctuaciones en el ambiente, durante el período de 
Tasa relativa de crecimiento

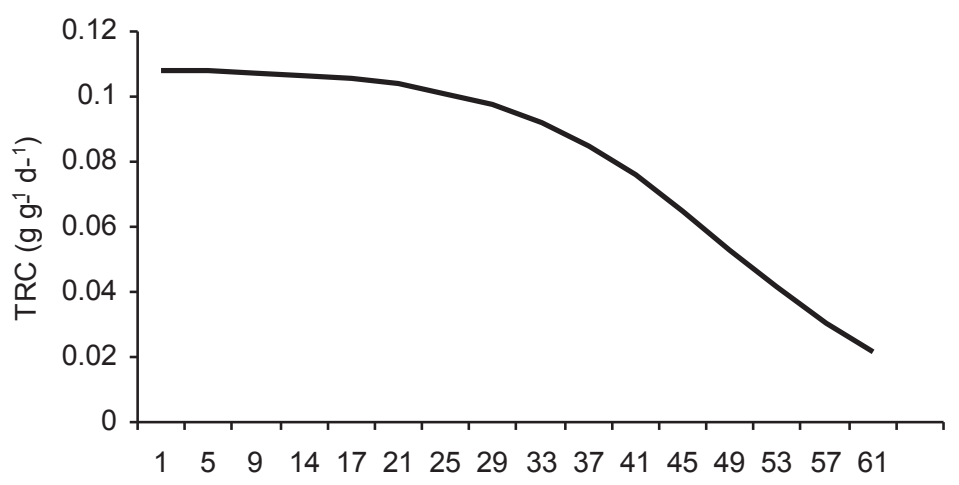

Días después de transplante

TRC

A.

Índice de área foliar

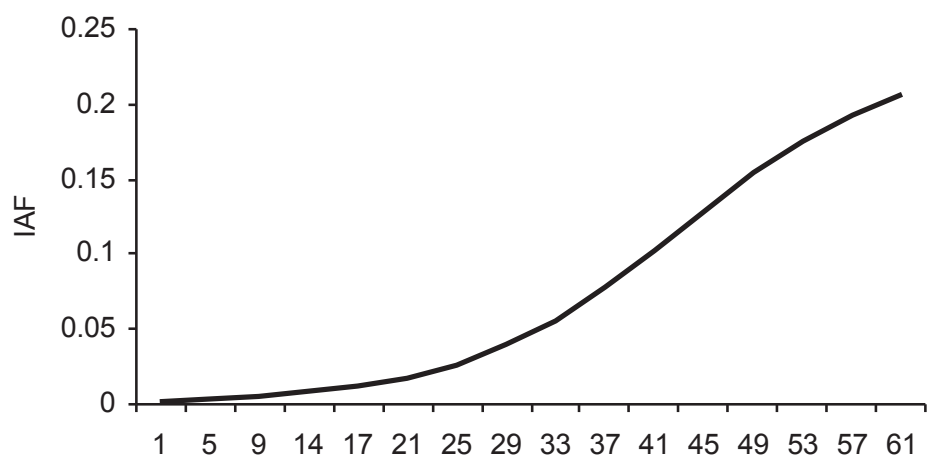

Días después de transplante

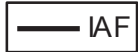

B.

Tasa de asimilación neta

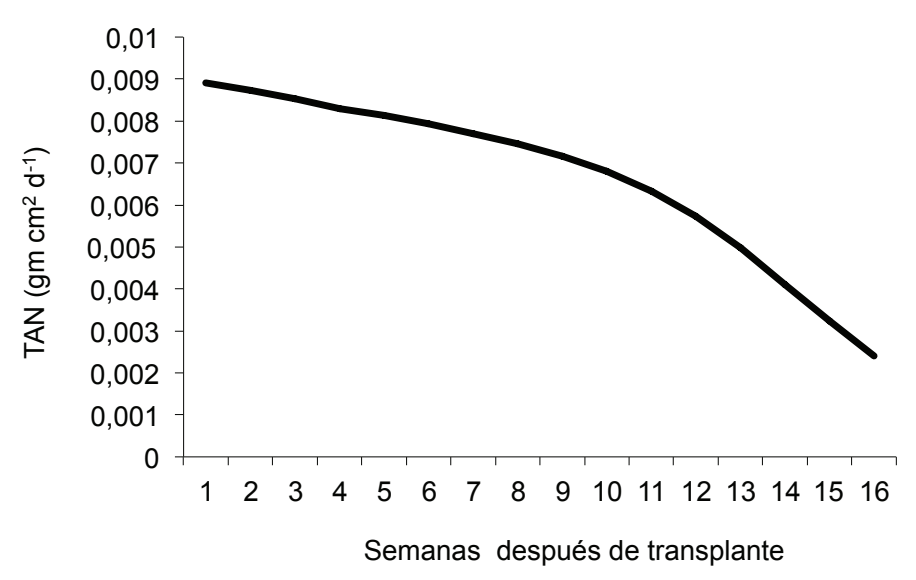

$\longrightarrow$ TAN

C.

Gráfica 2. Parámetros de crecimiento de la planta de albahaca. A. Tasa relativa de crecimiento (TRC); B. Índice de área foliar (IAF); C. Tasa de asimilación neta (TAN). 
tiempo analizado; de otra parte, Brown (1984) afirma que la TAN decrece con la edad de la planta, resultando en bajas eficiencias fotosintéticas. Carranza et al. (2009), encontraron para lechuga sembrada bajo un suelo salino un valor máximo de TAN de $0,00046 \mathrm{~g} \mathrm{~cm}^{2} \mathrm{~d}^{-1}$, a los $24 \mathrm{ddt}$, para luego empezar a decrecer.

Fases de desarrollo: Barroso \& Jerez (2002), trabajando con diferentes fechas de siembra de albahaca en un suelo ferrolítico de Cuba, determinaron seis fases: a) siembra, b) emisión del primer par de hojas verdaderas, c) emisión del primer par de ramas en las yemas laterales, d) aparición de la inflorescencia en el tallo central, e) aparición de la inflorescencia en el primer par de ramas laterales y f) senescencia del primer par de hojas. Contreras \& Gómez (2008) sugirieron cuatro fases para su trabajo: a) adaptación, b) crecimiento, c) aumento de biomasa y d) producción (prefloración). La caracterización de las fases de desarrollo, se sintetiza en la tabla 1 y se describe a continuación.

Fase 1. Esta primera fase va desde el momento de transplante, los 14 días después de transplante, donde la planta de albahaca inicia su crecimiento, acorde a la curva sigmoide, descrita por Salisbury \& Ross (1994), crecimiento lento, constante y positivo. Los parámetros de crecimiento indicaron los siguientes valores: TRC de $0,1064 \mathrm{~g} \mathrm{~g}^{-1} \mathrm{~d}^{-1}$, TAN de $0,0083 \mathrm{~g} \mathrm{~cm}^{2} \mathrm{~d}^{-1}$ e IAF de 0,0082 .

Fase 2. Va desde el día 15 hasta el día 31 después de transplantada la planta de albahaca; se caracteriza por un mayor crecimiento en sus variables: altura, número de hojas, peso fresco y área foliar. Los parámetros de crecimiento indicaron los siguientes valores: TRC de $0,092 \mathrm{~g} \mathrm{~g}^{-1} \mathrm{~d}^{-1}$, TAN de $0,0071 \mathrm{~g} \mathrm{~cm}^{2} \mathrm{~d}^{-1}$ e IAF de 0,047 . Esta fase se caracterizó por mayores variaciones en las variables de crecimiento, con respecto a la fase anterior.

Fase 3. La tercera fase empieza a partir del día 31 después de transplante y culmina bajo las condiciones del ensayo, el día 61 después de transplante. Esta fase, se caracteriza por un incremento importante en la altura y el número de hojas de la planta, como se muestra en la tabla 1; el peso de la planta también se incrementó de forma importante, al igual que el área foliar. Los parámetros de crecimiento indicaron los siguientes valores: TRC de $0,022 \mathrm{~g} \mathrm{~g}^{-1} \mathrm{~d}^{-1}$, TAN de $0,0024 \mathrm{~g}$ $\mathrm{cm}^{2} \mathrm{~d}^{-1}$ e IAF de 0,206.

Los valores de estos parámetros (TRC y TAN) indican una mayor eficiencia fisiológica de la planta de albahaca en la fase uno, como se puede apreciar en la tabla 1, eficiencia que va disminuyendo con el incremento del IAF de la planta, como se puede observar en las gráficas $2 \mathrm{~A}, 2 \mathrm{~B}$ y $2 \mathrm{C}$. Trabajo realizado por Carranza et al. (2009) mostró resultados similares en lechuga, en un suelo salino de la Sabana de Bogotá.
El mejor desempeño fisiológico de la planta de albahaca se manifestó durante los primeros $14 \mathrm{ddt}$, para ir decreciendo hasta los 61ddt, como consecuencia de un crecimiento sigmoidal característico; crecimiento controlado que, en un momento dado, puede variar por la interacción de la planta con el medio ambiente y por el manejo que se le de al cultivo. Se concluye que el productor debe centrar su atención al cultivo durante los primeros $14 \mathrm{ddt}$, período donde la planta debe llegar a maximizar su crecimiento y que las prácticas agronómicas, como variedad, nutrición, despuntes y riego, al igual que la siembra del cultivo en zonas cálidas, deberían cambiar los valores de crecimiento de la albahaca aquí presentados.

Se determinaron tres fases de desarrollo hasta llegar a floración, bajo las condiciones del trabajo, caracterizadas por cambios importantes en las variables evaluadas y en las tasas calculadas. Estas fases deben cambiar en los valores obtenidos en zonas cálidas, como el Valle del Cauca y Tolima, donde el ciclo del cultivo llega a los 48 - 50 días y el despunte que se le realiza al cultivo modifica la estructura de la planta y la eficiencia fisiológica de la misma; sin embargo, la información aquí expresada es útil, como referente para futuras investigaciones y para el cultivador de albahaca, ya que permite conocer el comportamiento del cultivo, a unas condiciones dadas de manejo y de ambiente.

Conflicto de intereses: El trabajo fue preparado y revisado por el primer autor, con el aval de los otros autores; no existe ningún conflicto de intereses que pongan en riesgo la validez de los resultados presentados. Financiación: Esta investigación fue financiada por la Universidad de Ciencias Aplicadas y Ambientales U.D.C.A.

\section{BIBLIOGRAFÍA}

1. ABELLO, J.; CLAVIJO, J.; BAREÑO, P. 2006. Estudio preliminar de algunos descriptores fisiológicos en cinco hierbas aromáticas. En: Últimas tendencias en hierbas aromáticas culinarias para exportación en fresco. Curso de extensión. Universidad Nacional de Colombia. Producción Editorial Produmedios. p.13-15.

2. AGUILAR, M.; CARRILLO, S.; RIVERA, A.; GONZÁLEZ, V. 2006. Análisis de crecimiento y de relaciones fuentedemanda en dos variedades de papa (Solanum tuberosum L.). Rev. Fitotecn. Mex. A.C. 29(2):145156.

3. BARACALDO, A.; IBAGUÉ, A.; FLÓREZ, V. 2010. Tasas e índices de crecimiento a segundo pico de cosecha en clavel estándar cv. Nelson cultivado en suelo y en sustratos. Agr. Col. 28(2):209-217. 
4. BARCELÓ, J., G NICOLÁS, G.; SABATER, B.; SÁNCHEZ, R. 1995. Fisiología Vegetal. Madrid, España. Ediciones Pirámide. 662p.

5. BARRAZA, F.; FISCHER, G.; CARDONA, C. 2004. Estudio del proceso de crecimiento del cultivo del tomate (Licopersicum sculentum Mill.) en el valle del Sinú medio, Colombia. Agr. Col. 22(1):81-90.

6. BARREIRO, A.; ZUCARELI, V.; ORIKA, E.; DOMINGOS, J. 2006. Análise de crescimiento de plantas de manjericáo tratadas com reguladores vegetais. Bragantia. 65(4):563-567

7. BARROSO, L.; JEREZ, E. 2002. Fenología de la albahaca blanca (Ocimum basilicum $\mathrm{L}$ ) cultivada en diferentes fechas de siembra. Cultivos Tropicales. 23(2):43-46

8. BERDONCES, J. 2010. Gran Enciclopedia de las Plantas Medicinales. De la A a la Z. Tomo 1. Grupo Océano, España, 646p.

9. BROWN, R.H. 1984. Growth of the green plant. En: Physiological Basis of Crop Growth and Development. Am. Soc. Agr. Madison. p.153-174.

10. CAMPOSTRINI, E.; YAMANISHI, O.K. 2001. Estimativa da área foliar do mamoeiro utilizando o comprimento da nervadura central. Sci. Agr. (Brasil). 58(1):39-42.

11. CARRANZA, C.; LANCHERO, O.; MIRANDA, D.; CHAVES, B. 2009. Análisis de crecimiento de lechuga (Lactuca sativa L.) "Batavia" cultivada en un suelo salino de la Sabana de Bogotá. Agr. Col. 27(1):41-48.

12. CASIERRA-POSADA, F.; HERNÁNDEZ, D.I.; LÜDDERS, P.; EBERT, G. 2003. Crecimiento de frutos y ramas de manzano Anna (Malus domestica Borkh.) cultivado en los altiplanos colombianos. Agr. Col. 21(1-2):6974.

13. CASIERRA, F.; CARDOZO, M. 2009. Análisis básico del crecimiento en frutos de tomate (Licopersicon esculentum Mill cv. "Quindio") cultivados a campo abierto. Rev. Fac. Nac. de Agr. Medellín. 62(1):48154822

14. CASTRO, R. 2005. Algunas notas sobre el cultivo de albahaca en un sistema hidropónico bajo invernadero, California USA. En: Hierbas Aromáticas culinarias para exportación en fresco. Curso de extensión. Universidad Nacional de Colombia. Facultad de Agronomía. Proyecto Hierbas Aromáticas. 15p.
15. CONTRERAS, A.; GÓMEZ, C. 2008. Evaluación de tres variedades de albahaca y dos dosis de fertilización en producción hidropónica y en suelo. Carrera de Ciencia y producción Agropecuaria. Zamorano, Honduras. 13p.

16. CORPORACIÓN COLOMBIA INTERNACIONAL CCI. 2007. Albahaca. En: Plan Hortícola Nacional. p. 223-232.

17. DONG, B.; SANG, W.L.; JIANG, X.; ZHOU, J.M.; KONG, F.X.; HU WANG, L.S. 2002. Effects of aluminum on physiological metabolism and antioxidant system of wheat (Triticum aestivum L.). Chemosph. 47(1):87-92.

18. FLÓREZ, V.; MIRANDA, D.; CHAVES, B.; CHAPARRO, L.; CÁRDENAS, C.; FARIAS, A. 2006. Parámetros considerados en el análisis de crecimiento en rosa y clavel en los sistemas de cultivo en suelo y sustrato. En: Flórez, V.J.; Fernández, A.; Miranda, D.L.; Chaves, B.C.; Guzmán, J.M. (eds.) Avances sobre fertirriego en la floricultura colombiana. UNIBIBLOS. p.43-52.

19. GHAZI, M.; NOBAHAR, A.; ALÍ, M. 2012. Effects of variety, planting pattern and density of plant phenology traits basil plants (Ocimum basilicum L.). Int. J. Agr. Crop Sci. 4(17):1221-1227

20. GRIME, J.P.; MACKEY, L.P. 2002. The role of plasticity in resource capture by plants. Evol. Ecol. 16:299-307.

21. HUNT, R. 1982. Plant growth curves. The functional approach to plant growth analysis. Edward Arnold Publishers, London. 248p.

22. HUNT, R. 2003. Plant growth analysis: individual plants. En: Thomas, B.; Murphy, D.J.; Murray, D. (eds). Encyclopaedia of Applied Plant Sciences. Academic Press, London, p.579-588

23. JARMA, A.; RENGIFO, T; ARAMÉNDIZ-TATIS, H. 2006. Fisiología de estevia (Stevia rebaudiana) en función de la radiación en el Caribe Colombiano. II. Análisis de crecimiento. Agr. Col. 24(1):38-47.

24. MALDONADO, G.; CORCHUELO, G. 1993. Dinámica de crecimiento de dos variedades de frijol (Phaseolus vulgaris L.). I. Análisis de crecimiento por períodos fijos. Agr. Col. 10(2):114-121.

25. MATUS, F.; MAIRE, C. 2000. Relación entre la materia orgánica del suelo, textura del suelo y tasas de mineralización de carbono y nitrógeno. Agr. Téc. 60(2):112-126. 
26. RAMÍREZ, J.;MORENO, A.; MURILLO, E. 2001. Evaluación agronómica e industrial de siete variedades de albahaca en la zona cafetera colombiana. Cenicafé. 52(2):117-126.

27. RUÍZ, F.; MARRERO, P.; CRUZ, O.; MURILLO, B.; GARCÍA, J. 2008. Influencia de los factores agroclimáticos en la productividad de albahaca (Ocimum basilicum
L.) en una zona árida de Baja California sur, México. Rev. Ciencias Téc. Agrop. 17(1):44-47

28. SALISBURY, F.B.; ROSS, C.W. 1994. Fisiología Vegetal. Grupo Editorial Iberoaméricana S.A. Mexico, 759p.

Recibido: Enero 24 de 2012

Aceptado: Marzo 25 de 2013 